\title{
CATEGORIZATION OF COMPENSATORY MOTIONS IN TRANSRADIAL MYOELECTRIC PROSTHESIS USERS
}

\author{
Ali Hussaini', Arthur Zinck' and Peter Kyberd ${ }^{2}$ \\ I Institute of Biomedical Engineering (IBME), University of New Brunswick, Fredericton, NB, Canada \\ 2 Department of Engineering Science, University of Greenwich, Chatham, Kent, UK, formerly Institute of Biomedical Engineering \\ (IBME), University of New Brunswick, Fredericton, NB, Canada
}

\begin{abstract}
Background: Prosthesis users perform various compensatory motions to accommodate for the loss of the hand and wrist as well as the reduced functionality of a prosthetic hand.

Objectives: Investigate different compensation strategies that are performed by prosthesis users.

Study Design: Comparative analysis

Methods: 20 able-bodied subjects and 4 prosthesis users performed a set of bimanual activities. Movements of the trunk and head were recorded using a motion capture system, and a digital video recorder. Clinical motion angles were calculated to assess the compensatory motions made by the prosthesis users. The video recording also assisted in visually identifying the compensations.

Results: Compensatory motions by the prosthesis users were evident in the tasks performed (slicing and stirring activities) as compared to the benchmark of able-bodied subjects. Compensations took the form of a measured increase in range of motion, an observed adoption of a new posture during task execution, and pre-positioning of items in the workspace prior to initiating a given task.

Conclusion: Compensatory motions were performed by prosthesis users during the selected tasks. These can be categorized into three different types of compensations.
\end{abstract}

\section{Clinical relevance}

Proper identification and classification of compensatory motions performed by prosthesis users into three distinct forms allows clinicians and researchers to accurately identify and quantify movement. It will assist in evaluating new prosthetic interventions by providing distinct terminology that is easily understood and can be shared between research institutions.

\section{Keywords}

Biomechanics, biomechanics of prosthetic/orthotic devices, motion analysis, myoelectric, prosthetics, rehabilitation, rehabilitation, rehabilitation of amputees, rehabilitation of prostheses users, transradial, unilateral, upper limb

prosthetics

Date received: 16 June 2015; accepted: 12 May 2016 


\section{Background}

Compensatory motions are the unusual or different movements performed by a person with physical or neurological limitations when they are attempting to complete a task. These differ from the usual observable motions of an unimpaired individual. For a given task, compensatory motions accommodate for the decreased functionality resulting from limb absence or amputation. However, repeated motions outside the typical range of the joint can increase stress of the muscle and joint and can increase the potential for an over use injury, such as a repetitive strain injury (RSI).'

The majority of studies on RSI have been focused on other activities, unrelated to prosthesis use. ${ }^{2-4}$ There has been little work, so far, in studying the effect of compensatory motions of prosthesis users and its long term implications for RSI. Based on a survey of subjects with amputations, Jones et al. ${ }^{5}$ concluded that there was a $50 \%$ chance that the subject would develop problems related to overuse. These symptoms were in the contra-lateral arm.

To improve patient functional assessment, awkward postures and movements need to be identified. In the study by $A t k i n s^{6}$, survey respondents indicated they needed (or wanted) to position their terminal devices by rotating the passive wrist to accomplish certain tasks. This suggests that compensations occur early in the task execution. Without a flexible wrist, the user has to find other ways to position their terminal device during the task, and it is expected that it will result in different forms of compensation. The objective of this study was to identify and define these compensation mechanisms.

This has two benefits: the proper identification of compensatory motions in prosthesis users may aid in the development of new prosthetic devices by increasing the understanding of how and why the motions are necessary with existing designs. Secondly, identifying the characteristic motions of this patient group may help in their recognition in individuals and lead to earlier intervention that may mitigate risk factors that lead to excessive strain and other injuries. ${ }^{7}$

The wrist plays a role in positioning the hand in space so that it may grasp the target object in a way that allows the task to be performed in the simplest and easiest manner. With few prosthetic wrist designs in common use, the literature on any wrist design is limited. The loss of the anatomical wrist limits the arm's motion and forces the user to correct for this limitation. There have been studies that have investigated the impact of limitations on the natural wrist and how it changes the way the arm is used. Studies performed by Ross $^{8}$ and MacPhee ${ }^{9}$ investigated kinematic motions of paediatric users with transradial prostheses when using two types of prosthetic wrist units. The first prosthetic wrist offered motion in one plane (pro-supination). The second wrist unit added an additional axis of rotation. Ross' study showed an increased in shoulder height of the healthy 
upper limb of unilateral prosthesis users when subjects were asked to zip up a vest. They also held this shoulder higher for a longer period of time as compared to the able-bodied group, (who did not show an appreciable difference in shoulder height). The multi-axis wrist reduced the height of the elbow as compared to the single-axis wrist, which suggests that more degrees of freedom can reduce the compensation in other joints. The study by MacPhee concluded that an additional degree of freedom in the wrist allowed for better positioning of the prosthetic hand relative to the target object, which resulted in kinematic movement that more closely resembled an able-bodied individual.

Gillen et al. ${ }^{10}$ looked at how a wrist should be splinted while the individual is recovering from a fracture. They concluded that 10 to 30 degrees of extension was important to provide function. This is in fact in variance to standard prosthetics practice that places the wrist in flexion, although this reflects the difference in the sort of activities the natural and prosthetic hand are used for. Mell et al." sought to see the impact of wrist splints on shoulder kinematics by asking subjects to pick up a ball from a box and place it in a tube. They found that the splinted subjects placed the arm above the box to use the elbow to reach down upon the target object. The reduction in range of motion at the wrist resulted in an increase in compensation at other limb segments.
There have been few previous studies on the motions of upper limb prosthesis users as they relate to compensations. Two studies have demonstrated that compensatory motions do occur when performing certain activities. Metzger et al. ${ }^{12}$ identified larger motions in the trunk for patients with an acquired transradial amputation when compared to a control group of able-bodied individuals. An earlier study by Carey et al. ${ }^{13}$ showed similar results for compensation in the trunk for myoelectric prosthesis users as well.

A study by Stavdahl' ${ }^{14}$ used able-bodied subjects with immobilizing cast to simulate the limitations of a prosthesis to study the compensations individuals used when a degree of freedom was removed. Stavdahl showed that compensations exist for able-bodied subjects when the degrees of freedom are restricted in the elbow and hand. This current study used a subset of Stavdahl's bimanual tasks for the subjects to perform. These tasks are simulated Activities of Daily Living (ADL). When comparing different subjects, it was important to pick activities that were familiar to all of the subjects. For example, raising a glass of water to the mouth can be considered a common activity that many would be familiar with. Operating a power drill may not be as familiar, but may be considered if the person works in a profession where this is common practice. 


\section{Methods}

Six bimanual ADLs were chosen to form the activity set for this study. They were selected from the Stavdahl set by the Occupational Therapist at the Atlantic Clinic for Upper Limb Prosthetics., to create the most effective set of representative tasks. The intention was to reflect as representative a set of activities as practical. These ADLs would also necessitate the use of the prosthesis, so that if any irregular motions (compensations) occurred, they would be seen immediately. A description of each is provided below:

Hanging clothes

The subject stands and uses clothespins to fasten clothing (shirts, socks, sweaters) placed on a table at waist height, onto a clothesline located $1.7 \mathrm{~m}$ above the floor.

\section{Slicing}

The subject stands at a table with a loaf of bread in line with the centre of the body. The subject holds the loaf of bread with one hand and is instructed to use a knife to cut a slice of bread approximately $2 \mathrm{~cm}$ thick.

\section{Stirring}

The subject stands at a table, with a round pot and stirring utensil placed on the table. The subject is instructed to grasp the pot at the rim and begin stirring the utensil inside the pot.

\section{Sweeping}

The subject stands and sweeps simulated debris into a pile using a broom.

\section{Eating}

The subject sits on a chair at a table with a knife, fork, and a slice of bread positioned in a plate on the table. The subject is instructed to cut a piece of the bread and eat the cut piece.

\section{Cutting}

The subject sits on a chair and holds a sheet of A4 size paper with their prosthesis. A pair of scissors are grasped in the other hand and used to cut a large circle from the paper.

For each task the subjects were allowed to perform the task in any way they chose, with either hand taking the dominant role.

Subjects for this experiment included 20 able-bodied subjects ( 13 male and 7 female) and 4 transradial prosthesis users ( 3 female, I male, all congenital absences). These were divided into two groups: right hand dominant and left hand dominant subjects. Three right hand dominant and one left hand dominant prosthesis users made up the patient group. Regardless of side dominance prior to the amputation, a patient's sound limb becomes their dominant side as it has more dexterity and more function then the side bearing the prosthesis. The prosthesis functions in a support role. All patients had a transradial prosthesis socket (supracondylar suspension) with a single degree of freedom electric hand. 
Right hand dominant prosthesis users employed an Otto Bock System Electric hand with either Twin digital or DMC control. The left hand dominant prosthesis user used an Otto Bock Trancarpal hand (Digital Twin Control).

Able-bodied subjects were recruited among students and faculty from the Mechanical Engineering and Nursing departments at the University of New Brunswick (UNB).[AQ: 4] Prosthesis users were clients of the Atlantic Clinic for Upper Limb Prosthetics. Involvement

in the study was voluntary. This study protocol received ethical approval from the UNB Research Ethics Board (REB 2006-I16). All subjects provided written informed consent for participation. All subjects completed the entire test procedure in a single visit to the Institute of Biomedical Engineering (IBME).

Details of the experiment and activities were explained and reflective markers were then attached to the subjects.

Motion capture sessions were performed at the IBME's motion analysis lab. The lab contains an 8 camera Vicon MCam system [Oxford Metrics, Oxford, England] which tracked the positions of reflective spherical markers attached to the subjects. Motion data were recorded at 60 $\mathrm{Hz}$. Trials were also recorded with a digital video recorder.

Markers used in this experiment were spherical and of two diameters. The smaller marker size was used when the distance to the nearest marker was relatively small. This ensured that the motion capture system could distinguish between the two locations. The anatomical location, size, and acronyms for the markers are listed in Table I. With prosthesis users, markers that were placed on the prosthesis were at locations similar to their anatomical equivalents, though a level of ambiguity is introduced as specific boney prominences do not exist. Three complete trials were recorded per activity and of these a single trial was chosen based on whether the recorded marker trajectory confused any markers or if there were missing markers in the capture volume.

\begin{tabular}{lll}
\hline Acronym & \multicolumn{1}{c}{ Anatomical Landmark } & Diameter (mm) \\
FRHD & Front of the head & 25 \\
\hline NECK & C7 of the Spine & 25 \\
\hline SACR & Flat part of the sacrum & 25 \\
\hline LTHD & Left side of the Head & 25 \\
\hline LCLA & Left sternoclavicular & 16 \\
\hline LSHO & Left shoulder marker & 25 \\
\hline LUPA & Left upper arm & 25 \\
\hline LELB & Left elbow lateral epicondyle & 25 \\
\hline LRAD & Left near left styloid process of radius & 25 \\
\hline LULNA & Left near left styloid process of ulna & 25 \\
\hline LWC & Left wrist centre & 16 \\
\hline L2MC & Left 2nd metacarpal head & 16 \\
\hline L5MC & Left 5th metacarpal head & 16 \\
\hline LHIP & Left front of ASIS & 25 \\
\hline RTHD & Right side of the Head & 25 \\
\hline RCLA & Right sternoclavicular & 16 \\
\hline RSHO & Right shoulder marker & 25 \\
\hline RUPA & Right upper arm & 25 \\
\hline RELB & Right elbow lateral epicondyle & 25 \\
\hline RRAD & Right near right styloid process of radius & 25 \\
\hline RULNA & Right near right styloid process of ulna & 25 \\
\hline RWC & Right wrist centre & 16 \\
\hline R2MC & Right 2nd metacarpal head & 16 \\
\hline R5MC & Right 5th metacarpal head & 16 \\
\hline RHIP & Right front of ASIS & 25 \\
\hline
\end{tabular}

Table 1 Subject Marker List

\section{Data Processing}

Using a subset of the 25 markers, 3 local coordinate systems were created to represent the pelvis, the trunk, and the head of the subject. Each coordinate system consisted of 
a set of 3 axes, which were created using the position data of the markers. To define the coordinate system, 3 noncollinear markers were used to create 3 directional vectors for the coordinate system. For this study, the clinically relevant angles as developed by $\mathrm{MacPhee}^{4}$ were employed. The clinically relevant angles were calculated from the appropriate coordinate systems, instead of the more familiar Euler or Cardan coordinate systems.

Three independent rotations were obtained by analyzing the trunk coordinates system relative to the pelvis coordinate system: lateral tilt, trunk (abdominal) flexion, and rotation (Figure I).
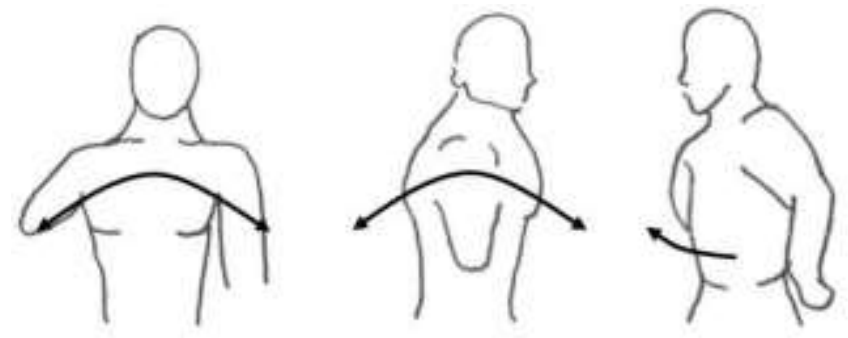

Figure 1. Trunk lateral tilt (left), trunk flexion (centre), trunk rotation (right)
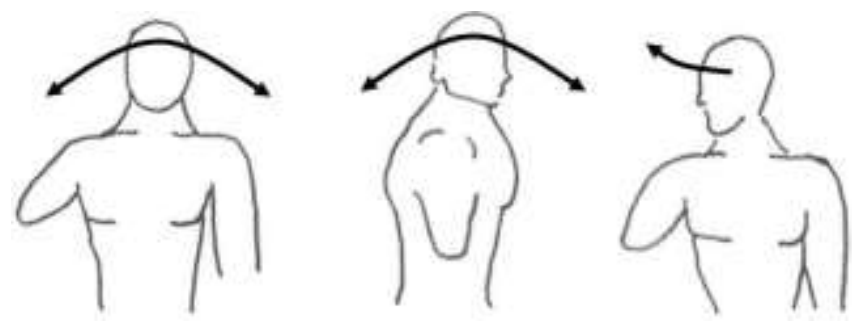

Figure 2. Head lateral tilt (left), head flexion (centre), head rotation (right)
Three rotations were obtained by analyzing the head coordinate systems relative to the trunk: lateral tilt, head flexion, and rotation (Figure 2).

For the pelvis and trunk, the $\mathrm{X}$-axis was directed forward, the Z-axis was directed upward, and the $\mathrm{Y}$-axis was to the subject's left-side. To define trunk lateral tilt, the line of intersection between the $\mathrm{YZ}$ plane of the pelvic coordinate system and the $X Y$ plane of the trunk coordinate system was found. The angle between the line of intersection and the $\mathrm{Y}$-axis of the pelvic coordinate system represented the lateral tilt.

Trunk flexion was defined by the line of intersection of the $X Z$ plane of the pelvis and the $X Y$ plane of the trunk coordinate system. The angle it made with the $\mathrm{X}$-axis of the pelvic coordinate system represents the trunk flexion.

Trunk rotation was calculated by creating a vector resulting from the cross product between the Z-axis of both the pelvis and trunk coordinate systems. The trunk coordinate system was then rotated about the new vector until the two Z-axes were aligned, making the $X Y$ planes of both coordinate systems coplanar. The angle between the two $\mathrm{X}$-axes is calculated as the trunk rotation. The same process was followed to determine the rotations for the head coordinate system with respect to the trunk. A detailed discussion of how these angles were developed can be found in works of MacPhee ${ }^{8}$ and Zinck $^{7}$. 
Before comparisons between subjects could be made it was necessary to normalize the trajectory data to decrease the dependency on time. During motion capture, patients moved from a rest position, performed the task, and returned to the rest position. This allowed for discrete events to be used for processing the data. The trajectories were sampled and normalized temporally to create data sets with 1000 frames. The analysis is concerned with frames 200 to 800 of the normalized data sets $(20 \%$ to $80 \%$ of recording). This range was used to truncate the periods of inactivity at either end of the captured data. Through this process, it was possible to compare patients who complete the same tasks, albeit at different speeds.

Trajectories were exported from the motion capture system. These were then imported into Matlab and manipulated upon by custom functions that created relative coordinates systems and calculated motion angles.

The different motions were compared and only those motions of the prosthesis users that showed clear compensations compared to the able bodied group are shown. Both of these groups are presented on the same graph. Due to the occultation of reflective markers, not all trajectories were complete. This resulted in the data from only 3 of the 4 prosthesis users being sufficient to plot trajectories (2 right hand dominant, I left). For the ablebodied group, only 7 right hand dominant subjects and 2 left hand dominant subjects are included in the graphs below.
The vertical axis represents the rotation angle in degrees. The horizontal axes are the normalized data points. The positive direction for lateral tilt and rotation is towards the dominant side. Flexion is positive in the forward direction.

\section{Results}

Initial analysis of the data showed that the prosthesis users showed three types of compensation: change in range of motion, adoption of a different posture, and prepositioning of items in the workspace. The results are presented in these groups.

\section{Range of Motion Compensations}

The slicing activity (Figure 3 ) shows the lateral tilt of 2 prosthesis users and the 7 able-bodied subjects (right handed). The trajectories of the prosthesis users are identified by the thicker bold lines. In this particular plot, the prosthesis users experienced the largest lateral tilt in terms of the rotation angle and the most variation in this same angle.

The motions of head flexion and head rotation also show large changes in range of motion in the prosthesis users' trajectories, and minimal change in the able-bodied subjects.

\section{Posture Compensations}

Adoption of new postures were identified on video review of a subject performing a given task, and confirmed in observing relatively constant trajectories. Figure 4 presents the lateral tilt of the trunk and head of I prosthesis user and 

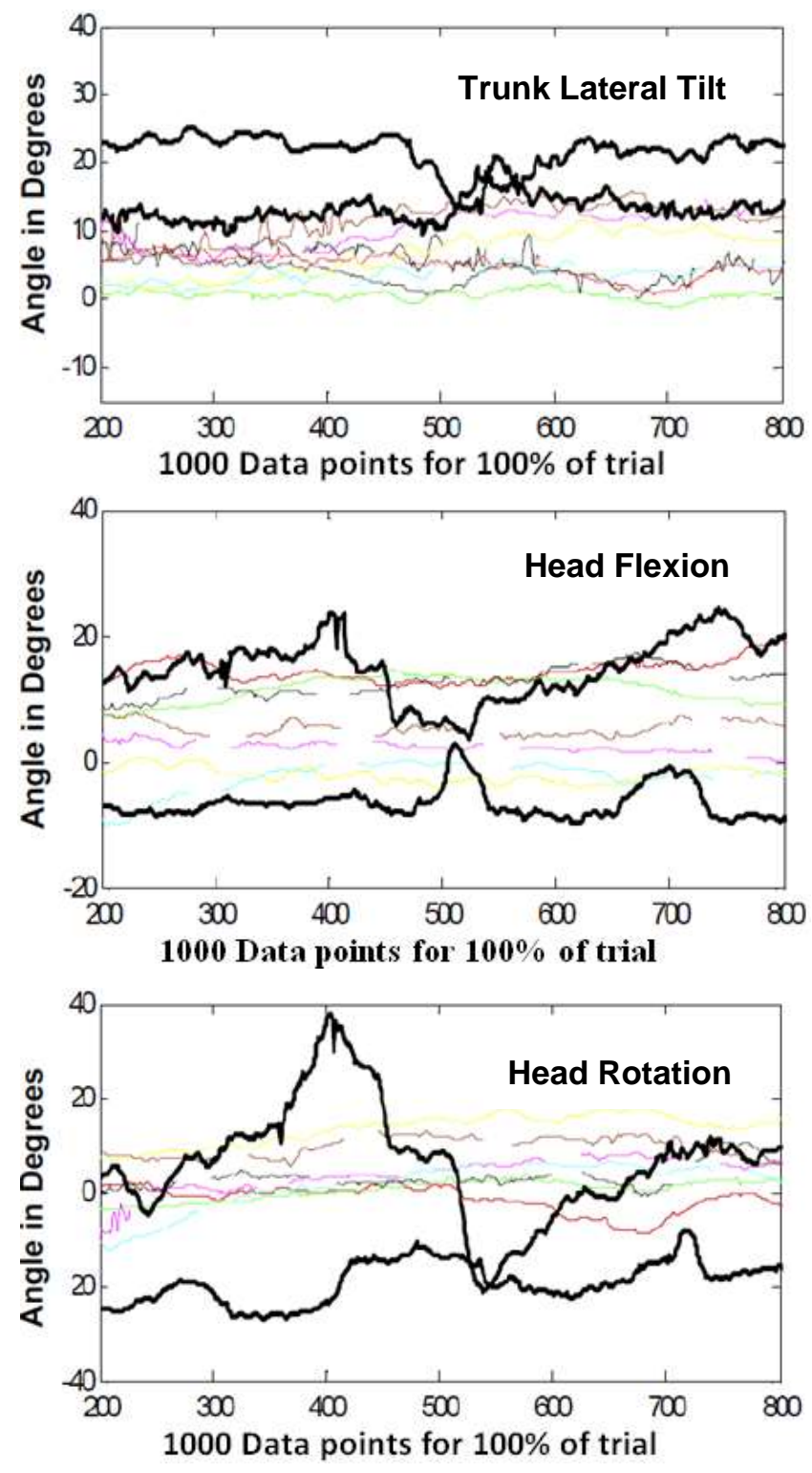

Figure 3.Slicing (right hand dominant subjects) with 2 prosthesis users in bolded line, 7 able-bodied in coloured. Prosthesis users experience largest range of motion and the most variation in both the lateral tilt and head flexion angles. Able-bodied subjects show minimal change during the task. The head rotation angle range of motion for one of the prosthesis users was approximately 60 degrees. The next highest range of motion belongs to the second prosthesis user.

2 able-bodied subjects (left hand dominant) performing the stirring activity. The prosthesis user begins the activity by adopting a trunk lateral tilt that is further towards their
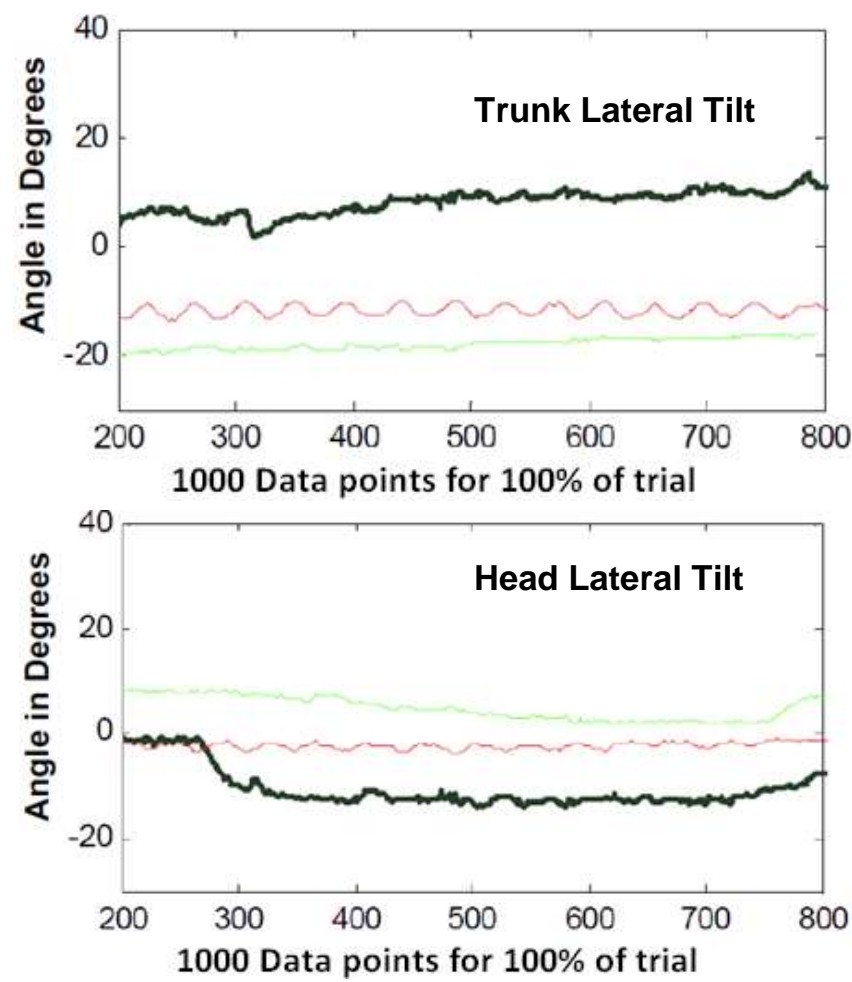

Figure 4. Stirring (left hand dominant subjects) with left hand dominant prosthesis user in bolded line, 2 ablebodied subjects in coloured. Prosthesis user adopts a trunk lateral tilt posture that is towards their dominant side and maintains this position. The user's head lateral

tilt posture is towards the non-dominant side (in opposition to their trunk lateral tilt). This is done to keep

the eyes focused on the task being performed.

dominant side and maintains this position for the duration of the activity. Figure 5 shows a captured video frame of an able-bodied user and a prosthesis user performing the stirring task to illustrate the difference in posture.

Lateral tilt of the head also shows a change in posture for the same activity of stirring. The prosthesis user began by adopting a head tilt towards their non-dominant side, and maintained this position for the duration of the activity. The other subjects also maintain their own selected head lateral tilt postures. 
Prepositioning of Items in Workspace

Prepositioning of items was seen on video review and was only present within the prosthesis user group. During the slicing activity, the prosthesis users concentrated on establishing a firm grip on the loaf of bread before initiating the slicing motion. The able-bodied patients performed these tasks simultaneously. In the stirring task, the prosthesis users began by ensuring that the rim of the pot was held securely in their prosthetic hand before the stirring action was performed. Similarly, in the eating activity the prosthesis users were observed positioning the fork in their prosthetic hand, ensuring a secure grip, and then proceeding to perform the activity. Again, the able-bodied patients were observed performing these motions simultaneously.

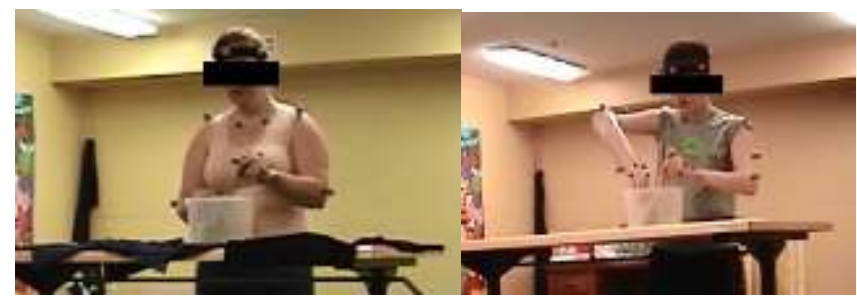

Figure 5. Stirring (left hand dominant subjects) with left hand dominant able-bodied subject (left) and prosthesis user (right). Prosthesis user adopts a trunk lateral tilt posture that is towards their dominant side. There is also an evident posture compensation on the prosthesis bearing side (elevated elbow) to keep the hand in position, grasping the rim firmly throughout the task.

It is important to note that the chosen ADLs require motions of the distal limb segments to complete the tasks as well. The range of motion of the more distal joints tended to be smaller than those of the trunk and the head, where compensations are more readily observed. However, additional compensatory motions were observed in both the shoulder and the humerus. During the slicing activity, an increase in shoulder flexion of the dominant arm and a noticeable change in range of motion in the elevation of the humerus of the non-dominant arm were seen with right hand dominant prosthesis users. In the eating activity, there was a noticeable increase in range of motion in the shoulder and elbow in the dominant arm for the left hand dominant prosthesis user. Trajectory plots for these and distal joints for all activities can be seen in the work by Zinck ${ }^{7}$.

Analyses of the hanging clothes, sweeping, and cutting tasks were not possible. In the hanging task, data for one of the prosthesis users was very poor and no motion was reconstructed. The clothing at times would block the view of motion cameras and became progressively worse as more clothes were hung (all subjects). A lot of the manipulation for this task occurred in the distal segments (wrist and hands), which were also blocked with more clothes on the line and when clothing was held in either hand. The use of the legs and feet in this task also made it difficult to gauge compensations. For upper limb tasks with motion capture, standing in one location is preferred, unless reflective markers are on the lower limbs as well. Otherwise, an excessive amount of variability in motion is introduced.

The sweeping task presented a similar issue. It was noticed that prosthesis users positioned the broom in front 
of themselves, instead of holding the broom off to one side as able-bodied subjects did. This could be seen as a posture compensation. Depending on whether subjects (able-bodied and users) held the broom to the left and right sides during sweeping, this also caused variability. Since the broom handle created a rigid linkage between the two hands, the contribution of each of the anatomical hand and the prosthesis was difficult to separate. Linked hand task like this should be avoided if the intention is to see the difference the prosthesis makes. Subjects were not instructed how to sweep the debris on the floor, just to do it. With no clear motion to follow, comparing the trajectories was questionable.

Cutting a circle out of a piece of paper had more variation between prosthesis users, and made it difficult to see whether a compensation existed. Some users used the scissors at times to hold the paper while the prosthesis was repositioned. Some rested their prosthesis on their lap so they did not have to hold the prosthesis up. This task resulted in many variations in body segments making it difficult to see (and show) compensations in one or two motions and required all the limb segments to be seen to appreciate.

\section{Discussion}

In order to classify and investigate the compensatory motions of patients with transradial amputations, this study proposes definitions of three compensatory strategies.
These strategies depend on the difference in the range of motions between the prosthesis users and a benchmark group (able-bodied subjects).

Different postures The first type of compensation involves the adoption of a new static posture (compared to the ablebodied subjects) to perform activities. The subject moves their body segment into a new position, which is then held constant throughout the exercise.

Range of motion The second category of compensation is that of an increase or decrease in range of motion of a joint or body segment. This refers to the change in rotation angle when compared to the benchmark group.

The first category differs from the second in that the adopted posture stays relatively constant throughout an activity. A range of motion compensation implies a moving trajectory of a body segment (a dynamic difference). This can be recorded by a change in trajectory of a marker in the motion capture system. Posture changes are observed through inspection of the action (e.g. video recording).

Prepositioning The third category of compensation is the prepositioning of items in the workspace. This happens before an activity is performed. For example, a fork can be positioned in the prosthetic hand by the intact hand before eating a meal.

The three compensations identified in this study suggest that when a patient utilizes their prosthesis, their approach to solving a given problem is multidimensional. However, 
there was a hierarchy to implementing the compensatory motions. The prepositioning of items was always executed before either of the other two compensations could be conducted. This makes intuitive sense as the user would assess their workspace and develop a strategy to complete the exercise. This is supported by the fact that the prepositioning of items only occurred when the item in the workspace was to be manipulated by the prosthesis and not the anatomical hand. This showed that the patient was compensating for the reduced dexterity of the prosthesis.

Prosthesis users adopted different postures for certain tasks, and the prepositioning of items in the workspace was far more evident when compared to the able-bodied group. As a result, it was difficult to properly compare the range of motion compensations and synergies in the motion of body segments with the other group. This is because the range of motion is influence by postures and the prepositioning of items which occur before a task is initiated. Postures and prepositioning of items was observed and/or confirmed after video of the trial was reviewed. This made it difficult to evaluate these compensations as a measured statistic. These are the primary compensations for prosthesis users, in the sense that they are the most evident forms and they, by definition, occur before the range of motion compensations can be measured. To rectify this, the chosen task must be controlled enough to eliminate or account for the need for prepositioning and posture adoption.
The measured rotation angle in a range of motion compensation varies with time. During the slicing activity the lateral tilt trajectory changes for all subjects, but the variations in the angles for the prosthesis users are more pronounced than the able-bodied users, in terms of lateral tilt of the trunk, head flexion, and head rotation. A similar trend was observed with the posture compensation in the stirring activity where the absolute values for lateral tilt of both the trunk and the head are larger for the prosthesis user.

The patients in this study all presented with a congenital limb absence and used single degree of freedom myoelectric devices. Metzger's study included myoelectric and body powered devices which introduced proprioception as a variable. From the results presented in that work, it is unclear what effect proprioception had on these compensation. A study that can distinguish between the roles of proprioception and simply additional degrees of freedom as it relates to compensatory motion may provide insight on the role of proprioception. The research question then posed would be whether the user changes the positioning/orientation of their hand (and by extension their body), if they can feel the grasped object. The results presented in this study do confirm the conclusions of the previous studies $^{12,13}$ that an increased compensation exists in the trunk for prosthesis users with transradial amputations. 
The trunk compensation is the result of an inability to position the terminal device. Providing a prosthesis user with additional degrees of freedom distally can reduce the compensation required at the trunk, as long as the user can make effective use of the range of motion. Advanced prosthetic designs should consider the reduction of compensatory motions as a primary objective. With the classification scheme proposed in this work, it was seen that prepositioning of the prosthetic hand can influence the other two categories of compensation. Therefore, an improved ability to preposition the hand through a powered wrist or elbow joint may be the appropriate avenue for future prosthetic designs, rather than to require the prosthesis to be aligned during manipulation.

Limitations in the study design and the functionality of the prosthesis should be noted. The restrictions in the flexibility of the grasp of the hand may impose upon the approach vector of the prosthesis to the object. Unlike a natural hand that can pick up an object in a range of different ways and angles, the prosthesis may be limited to one. This therefore may be driving all three forms of compensation.

Identifying a posture compensation can be difficult if the prosthesis user and able-bodied subjects are of different heights. When discussing posture compensations at the trunk and head, a degree of ambiguity is introduced. A taller person may show a larger head flexion angle to focus on the workspace. Matching the height of the experimental and control population may mitigate this effect. It must be noted that with a small patient population, which is the case in many upper limb prosthetic studies, it is often difficult to demonstrate consistency in the resulting compensations across the prosthesis users and a selection of able bodied subjects represents a sample of the many individual solutions chosen. The solutions adopted by other unimpaired subjects may also deviate from the methods observed. These deviations may be for societal or physiological limitations not considered in this study. The motions herein identified as compensations are those actions taken to overcome the limitations of the prostheses worn, even in a greater and more heterogeneous population they would describe the compensations used with the prostheses. Figure 3 (head flexion) reflects this problem with two users who both show different range of motion compensations. At data point 500, the direction of their trajectories are opposite, though both are performing the same task, and both have the prosthesis on the same side. Mitigation of this variability may be possible by choosing tasks that are not bimanual, and preventing the motion of the hand that is not in use.

\section{Conclusion}

Compensatory motions are undertaken by users who employ a transradial prosthesis. This study proposes a classification system that identifies three distinct forms: Prepositioning, Posture and Range of Motion and observes the hierarchy which the user employs each one. Though 
compensatory motion studies with upper limb prosthesis users have been performed, it is believed that this study is the first to classify compensations into different types.

\section{Acknowledgements}

The authors would like to thank the faculty of the Institute of Biomedical Engineering (IBME) at the University of New Brunswick for their assistance with the motion capture system and Krista Fraser and the clinical team at the Atlantic Clinic for Upper Limb Prosthetics in Fredericton for coordinating patient visits for this study.

\section{References}

[I] Kidd PS, McCoy C, Steenbergen L. Repetitive strain injuries in youths. J Am Acad Nurse Pract 2000; 12(10):413426.

[2] Fortenbaugh D, Fleisig GS, Andrews JR. Baseball pitching biomechanics in relation to injury risk and performance. Sports Health 2009; I (4):3 I4-320.

[3] Hansson et al. Physical workload in various types of work: Part I. Wrist and forearm. Int. J. Ind. Ergon 2009; 39(I):22I-233

[4] Muggleton JM, Allen R, Chappell PH. Hand and arm injuries associated with repetitive manual work in industry: a review of disorders, risk factors and preventive measures. Ergonomics 1999; 42(5): 714-739.

[5] Jones LE, Davidson JH. Save that arm: a study of problems in the remaining arm of unilateral upper limb amputees. Prosthet Orthot Int 1999; 23(1):55-58.
[6] Atkins, DI, Heard DCY, Donovan WH. Epidemiologic Overview of Individuals with Upper-Limb Loss and Their Reported Research Priorities. J Prosthet Orthot 1996: 2-II.

[7] Zinck A. Investigation of Compensatory Movements in Prosthesis Users and the Design of a Novel wrist. Master's Thesis, University of New Brunswick, 2008.

[8] Ross M. Development of a Quantitative Test for Prosthetic Function Using Motion Analysis and Activities of Daily Living. Master's Thesis, University of New Brunswick, 2005.

[9] MacPhee B. Examining the Prosthetic Function and Body Behaviour of Prosthesis Users Performing Activities of Daily Living. Master's Thesis, University of New Brunswick, 2007.

[10] Gillen G, Goldberg R, Muller R et al. The Effect of Wrist Position on Upper Extremity Function While Wearing a Wrist Immobilising Splint. J Prosthet Orthot 2008; 20(I):1923.

[II] Mell AG, Childress BL, Hughes RE. The effect of wearing a wrist splint on shoulder kinematics during object manipulation. Arch Phys Med Rehabil 2005; 86(8): I66I-I664.

[12] Metzger AJ, Dromerick AW, Holley RJ et al. Characterization of compensatory trunk movements during prosthetic upper limb reaching tasks. Arch Phys Med Rehabil 2012; 93(II):2029-2034.

[13] Carey SL, Highsmith MJ, Maitland ME et al. Compensatory movements of transradial prosthesis users during common tasks. Clin Biomech (Bristol, Avon) 2008; 23: I $128-35$.

[14] Stavdahl O. Optimal Wrist Prosthesis Kinematics: Threedimensional Rotation Statistics and Parameter Estimation. PhD Thesis, Norwegian University of Science and Technology, 2002. 